\title{
DESERCIÓN O EXCLUSIÓN ESCOLAR: ANÁLISIS SOBRE EDUCACIÓN, DESIGUALDAD Y POBREZA EN PUERTO RICO
}

\author{
Gloria Rosado Ortiz'
}

\section{Resumen}

La deserción escolar en Puerto Rico es una manifestación del vínculo entre educación, desigualdad y pobreza. El artículo devela los mecanismos en las políticas educativas actuales que reproducen la desigualdad educativa y la desigualdad social en Puerto Rico. Explica cómo esos mecanismos se manifiestan y examina su impacto en la niñez que vive en pobreza. La autora hace un recuento del estado de derechos de la niñez, con énfasis en el derecho a la educación. Destaca la relación entre Estado, sociedad, economía y educación en diferentes contextos históricos. Resume el trasfondo histórico de las políticas educativas para atender la desigualdad social en Puerto Rico y los Estados Unidos. Profundiza en el impacto en la niñez pobre, de la implantación de las actuales políticas educativas federales. [Descriptores: deserción escolar, pobreza, desigualdad, políticas educativas].

\section{Abstract}

Dropping out of school in Puerto Rico shows the link between education, inequality and poverty. The author reveals the current educational policies mechanisms reproducing social inequalities in Puerto Rico. Explains how those mechanisms occur and examine its impact for the childhood living in poverty. It summarizes the state of children rights, with an emphasis on

Candidata a grado doctoral en Análisis de Política Social y Administración de Programas Sociales, de la Escuela Graduada de Trabajo Social de la Universidad de Puerto Rico, Recinto de Río Piedras. 
the right for education. Focusing on the government, society and market relationship on various historical contexts. Summarizes the historical background of educational policies to alleviate social inequalities on Puerto Rico and the United States. Explores the implementation of the current federal educational policies and its impact on poor children.

[Keywords: dropout, poverty, inequality, educational policies].

\section{Introducción}

La deserción escolar es una manifestación del vínculo entre educación, desigualdad y pobreza. Las políticas educativas contribuyen a la reproducción de las desigualdades; ya que tienen una misión social, pero están diseñadas en función del mercado neoliberal. Existen elementos al exterior y al interior del sistema educativo público, que inciden en que una cantidad considerable de estudiantes en el nivel secundario no culmine el proceso educativo que los vincula a la futura fuerza laboral del país. Para el estudiantado de comunidades pobres, el rezago educativo y la limitación de sus futuras oportunidades de empleo, se dan en un contexto económico que cada vez exige mayor dominio académico y tecnológico para el empleo. De ahí que, parte de la niñez queda excluida del sistema de oportunidades socioeconómicas, lo que representa una violación de sus derechos humanos.

El artículo intenta develar los mecanismos en las políticas educativas actuales que reproducen la desigualdad educativa y social en Puerto Rico. Busca explicar cómo esos mecanismos se manifiestan y su impacto en la niñez pobre. Hace un recuento del estado de derechos de la niñez, con énfasis en el derecho a la educación. Destaca la relación entre Estado, sociedad, economía y educación en diferentes contextos históricos. Discute el trasfondo histórico de las políticas educativas para atender la desigualdad social en Puerto Rico y los Estados Unidos. Profundiza en el impacto en la niñez pobre, de la implantación de las actuales políticas educativas federales. Al final, plantea retos y recomendaciones para diversos sectores sociales, dirigidos a la prevención de la deserción escolar.

\section{Desigualdad, deserción escolar y derechos de la niñez}

En toda sociedad ha habido diferencias entre diversos sectores, en términos de la distribución de recursos, poder y oportunidades. Para Benaabdelaali, Hanchane y Kamal (2011), "la desigualdad es un concepto multidimensional, que incluye la 
distribución desigual de ingresos e inequidades en oportunidades sociales en salud, vivienda y educación, de unos sectores sociales con menos acceso a recursos y oportunidades de desarrollo" (p. 2). Para los sectoresque confrontan desigualdad, los riesgos sociales se profundizan, a la vez que toman nuevas y diversas formas. La niñez, de acuerdo a Minujim (2012):

... es un sector social muy vulnerable a la privación porque requiere de recursos y protecciones especiales para su subsistencia y desarrollo. Es por lo que la desigualdad tiene un impacto distinto en la niñez, con implicaciones a lo largo de la vida del ser humano. (p. 14)

Por eso es urgente la atención al impacto de la desigualdad y la pobreza en la niñez ya que tiene consecuencias en el presente y el futuro de sus vidas.

Los conceptos de desigualdad y exclusión social son una construcción social derivadas de condiciones económicas, sociales, políticas y culturales que componen un modelo de organización social. Para De Sousa (2003), ambos son sistemas de pertenencia jerarquizada, manifestados de distintas formas. En la desigualdad, la pertenencia se da por la integración subordinada, mientras que en la exclusión no hay integración². Sobre la desigualdad en Puerto Rico, se estima que el $20 \%$ de los hogares con ingreso promedio más alto, es 33 veces mayor que el $20 \%$ de los hogares con el ingreso promedio más bajo. "La relación de desigualdad en el promedio de ingreso de los hogares en Puerto Rico, es de 33 a 1 y contrasta con la relación que se da en los Estados Unidos, de 15 a 1" (Marxuach, 2012, p. 16). Siendo estos conceptos construcciones sociales, reflejan carencias como el hambre, que puede llevar a un ser humano hasta la muerte.

La desigualdad, la exclusión social y la pobreza han estado históricamente vinculadas. Rodríguez (2012) plantea un concepto abarcador de la pobreza, a la luz de las implicaciones

\footnotetext{
${ }^{2}$ La desigualdad implica un sistema jerárquico de integración social. Quien se encuentra abajo está adentro, y su presencia es indispensable. Por el contrario, la exclusión presupone un sistema igualmente jerárquico pero dominado por el principio de la exclusión: se pertenece por la forma como se es excluido. Quien está abajo, está afuera (De Sousa, 2011, p. 125).
} 
que le atribuye. Para la autora, la pobreza tiene implicaciones económicas, por la carencia de ingresos que la caracteriza; individuales, por la limitación que impone a la satisfacción de necesidades y al desarrollo de capacidades; sociales, por la marginalidad y la falta de participación que ocasiona; y para el bienestar humano, por la poca felicidad y calidad de vida que genera. Por espacio de las últimas décadas, en Puerto Rico más de la mitad de la niñez ha vivido por debajo del nivel pobreza. Annie E. Casey Foundation (AECF) (2012), establece que "para la niñez, el vivir en áreas de extrema pobreza, escasea y pone fuera de su alcance los recursos para el crecimiento saludable y el desarrollo, como escuelas con desempeño de calidad, servicios de salud de calidad y espacios seguros" (p. 1). AECF (2012) plantea que cuando en las comunidades aumenta el nivel de pobreza, con ello aumentan las condiciones adversas para el desarrollo de la niñez; y disminuyen sus oportunidades de éxito. De esa manera, no solo se violan los derechos humanos de la niñez que vive en pobreza, sino que la desigualdad se reproduce de una generación a otra.

AECF (2012) estima que el $83 \%$ de la niñez en Puerto Rico vive en comunidades que tienen $30 \%$ o más de sus residentes, viviendo por debajo del nivel de pobreza. La Puerto Rico Community Survey (PRCS, 2012) estima que, actualmente el $56.4 \%$ de la niñez en Puerto Rico vive en hogares de familias que se encuentran bajo el nivel de pobreza. El incremento de la pobreza ha afectado de manera desproporcional a la población menor de dieciocho años.

La niñez proveniente de comunidades y de familias que viven en pobreza, se encuentra más vulnerable, carente de los recursos y oportunidades necesarias para el pleno desarrollo y el potencial de un futuro de vida digna, con bienestar y calidad. Esto supondría que, de no intervenir el Estado, a largo plazo se reproducirá el ciclo de desigualdad para estas personas y sus familias, con efectos multiplicadores adversos para la gobernabilidad del país. Ese sector de la niñez confronta más obstáculos en su desarrollo educativo. La desigualdad de oportunidades educativas es definida como la menor posibilidad que tienen las personas pobres de ingresar a instituciones del sistema educativo, permanecer hasta terminar los ciclos escolares y lograr aprovechamiento escolar satisfactorio (Bazdresch, 2001). Los factores socioeconómicos inciden sobre la educación y el 
aprendizaje en áreas como: el acceso, la retención, los resultados del aprendizaje, la culminación del ciclo de educación formal y la posibilidad de un proceso de aprendizaje a lo largo de toda la vida. Esto implica que su proceso de formación educativa, su potencial desarrollo ocupacional y económico futuro, se verá impactado de manera adversa por la pobreza.

El Estado también puede generar problemas educativos en términos de calidad en los servicios, eficiencia en los procesos y los resultados; así como en la cobertura educativa para toda la población. Brussi (2010) hace referencia a las barreras institucionales, que el continuo escuela-universidad pone para que estudiantes de comunidades pobres en Puerto Rico, logren el acceso a estudios universitarios, imponiendo el currículo vocacional a estudiantes por su lugar de procedencia. La autora establece que "las propias instituciones educativas a veces imponen o refuerzan estructuras que dificultan el acceso y éxito universitario" (p. 2). De esta forma, se afecta la probabilidad de recibir un mensaje de expectativas universitarias y el rigor académico al que está expuesto el estudiantado de comunidades pobres, en su etapa preuniversitaria (Brussi, 2010). Añade Dieterich (1996) "que en los países latinoamericanos en desarrollo, la educación por sí sola no es una variable clave del desarrollo colectivo de la nación ni de su salida del subdesarrollo, aunque suele ser un instrumento de movilidad social" (p. 14). Estos autores denuncian unas políticas educativas que en su diseño e implantación, promueven la desigualdad educativa.

Considerada de esta forma, la deserción escolar podría analizarse como un fenómeno de exclusión escolar; o el resultado de políticas y prácticas educativas desiguales y excluyentes para el estudiantado proveniente de comunidades pobres. Según la PRCS (2012), se estima que en Puerto Rico, $29.6 \%$ de los hombres y el $25 \% 6$ de las mujeres de 25 años o más; así como el $15.6 \%$ de los hombres, y $10.9 \%$ de las mujeres entre 18 y 24 años, no completaron escuela superior. Estas últimas (18 a 24 años), con pocas oportunidades laborales, de desarrollo y bienestar, se estiman cerca de 51,283 personas.

La exclusión se transforma en riesgo con serias consecuencias. En el 2011, la Administración de Corrección de Puerto Rico ofreció un perfil de los 406 jóvenes institucionalizados con la siguiente información: $55 \%$ tenía una educación de octavo grado o menor, $65.2 \%$ tenía bajo aprovechamiento académico, 
$73.8 \%$ había tenido algún fracaso escolar en el nivel intermedio, y $63.6 \%$ había sido desertor escolar. La vulnerabilidad en la que se encuentra la niñez pobre en Puerto Rico, especialmente la población masculina, es de tal magnitud que se nos señala como uno de los países latinoamericanos con tasas más altas (41.8) de homicidio juvenil por cada 100,000 habitantes (Organización Panamericana de la Salud, 2003). El Center for Desease Control and Prevention (2005) estimó que entre el 1999 y el 2003, el homicidio fue la quinta causa de muerte para los varones y el $93 \%$ de las víctimas eran varones menores de 30 años en Puerto Rico. Estas consecuencias requieren del Departamento de Educación, una mirada reflexiva y autocrítica sobre su responsabilidad institucional con relación a la deserción escolar; más allá de fijar responsabilidades en el individuo y la familia.

Históricamente la niñez ha sido considerada como un sector sin voz ni derechos. Es por lo que el enfoque de derechos de la niñez que se promueve actualmente incluye aspectos relacionados a su protección, salud, educación y bienestar económico. Prioriza en la atención específica a sus necesidades y derechos como seres humanos, en la legislación y la implantación de políticas públicas. En la Convención de los Derechos del Niño de 1989, la Organización de Naciones Unidas estableció derechos específicos para la niñez. Sus propósitos fueron: el reconocimiento y defensa de la niñez como seres humanos sujetos de derechos; y llamar la atención sobre el estado de la niñez, de su particular experiencia, en términos de vulnerabilidades, intereses y garantías sociales (Redmond, 2008). La Convención, en el Artículo 28 establece "el derecho de la niñez a la educación, a fin de que se pueda ejercer progresivamente y en igualdad de oportunidades" (según citado en Redmond, 2008, p.12). El Inciso 1, establece los derechos para la niñez y la responsabilidad de los estados en términos de lo siguiente: enseñanza primaria, obligatoria y gratuita; fomentar el desarrollo de la enseñanza secundaria, general y profesional; hacer la enseñanza superior accesible; garantizar el acceso a información educativa y profesional; y adoptar medidas para fomentar la asistencia regular a la escuela y reducir las tasas de deserción escolar.

Actualmente, aumentan los reclamos para la atención de la desigualdad educativa en el mundo y en América Latina, crecen las voces por el acceso a una educación de calidad como medidas 
de protección social del Estado para atender la desigualdad y la vulnerabilidad de varios sectores poblacionales. No basta el acceso a educación gratuita, sino que debe garantizarse una educación de calidad para toda la niñez. Puerto Rico estuvo a la vanguardia en la promulgación de derechos educativos para toda la ciudadanía. La Constitución del Estado Libre Asociado de Puerto Rico (1952), garantiza en su preámbulo el "promover el bienestar general y asegurar para nosotros y nuestra posteridad el goce cabal de los derechos humanos" (p. 1). La Carta de Derechos en la Sección 5 reconoce "el derecho de toda persona a recibir gratuitamente la instrucción primaria y secundaria" (1952, p. 4). Por la Constitución, la agencia encargada de dictar política pública para la educación primaria y secundaria en Puerto Rico, es el Departamento de Instrucción Pública, actualmente Departamento de Educación (DE). Sin embargo, el DE no puede garantizarle hoy a toda la niñez, igualdad en la calidad de la educación pública.

\section{Estado, sociedad, mercado y educación}

La educación ha estado vinculada a la relación entre la sociedad, el Estado y el mercado. Bonilla (2004) plantea que "la escuela republicana o la educación popular de la etapa colonizadora de la historia de nuestros países latinoamericanos, es el origen de nuestros actuales sistemas educativos de masas" (p. 90). Sin embargo, aún dentro de esa escuela popular de carácter democrático, hubo exclusión de sectores sociales importantes como las comunidades originarias, las afrodescendientes, campesinas, personas con impedimentos, así como mujeres y niñas. Añade Bonilla (2004) que "a mediados del siglo XX, el modelo de la escuela republicana cumplió sus objetivos de educación universal, extensión del periodo escolar, creación de ciudadanía y elevación cultural de la nueva población o los sectores incorporados al proceso educativo, en función de las necesidades sociales y económicas de los países, en ese momento histórico" (p. 76). Esto supone nuevos retos en materia educativa para la sociedad actual impactada por el neoliberalismo.

En la actualidad, la relación entre sociedad, Estado y mercado ha experimentado un giro en el paradigma económico y social. Bauman (2005) plantea que el Estado benefactor, en la sociedad basada en la ética del trabajo, tenía la responsabilidad de atender el bienestar público y garantizar la digna supervivencia de las personas, para su incorporación y productividad dentro del 
mercado laboral. Mediante políticas universales de protección social, se transformó el derecho a una vida digna en una cuestión de ciudadanía, a la vez beneficiosa para la sociedad. Se creó una generación educada, saludable, segura de sí misma, de su independencia y con posibilidad de movilidad social. Por el contrario, la sociedad de consumo, basada en la estética del mercado, tiene al individuo consumidor como unidad de intervención. "Una sociedad donde la riqueza y el consumo se normalizan, convirtiéndose en fines en sí mismos; lo que contrasta con la ética del trabajo y el esfuerzo individual para el logro del bienestar individual y colectivo" (Bauman, 2005, p. 56). El Estado ha sufrido la disminución de su capacidad como generador de cambio social.

En la sociedad de consumo, la función social del Estado benefactor entra en contradicción con el mercado. Mientras este rinde culto a la diferencia y a la elección, el sentido del Estado se encuentra en la idea de la igualdad en la atención de necesidades y en la protección de derechos. En la economía neoliberal el Estado ve constantemente reducidos sus poderes y capacidades. Poderes que son reducidos en las medidas de protección social, en las estrategias para atender la pobreza y en la delimitación del tipo de población o de pobres a quienes se dirigen los servicios. Dicha situación pone al Estado "entre la inclusión y la exclusión" (Bauman, 2005, p. 76). La paradoja del Estado reside en el dilema de ¿a quién servir?

Si la pobreza es vista como innecesaria para el funcionamiento de la economía, el ejército de personas desempleadas que en la modernidad equilibraba al mercado laboral no representa nada para el mercado de consumo. Bauman (2005) describe que ser pobre en la sociedad actual es sinónimo de ser un consumidor defectuoso, frustrado y un excluido del mercado, por considerarse un estorbo. Como en el neoliberalismo, el mercado determina las reglas sociales, el estar fuera del mercado puede generar una progresiva exclusión social para el ser humano.

Si el Estado se debate entre dos fuerzas, la desigualdad social se acrecienta en detrimento de los sectores sociales más vulnerables. Para Ander-Egg (1995) y Colón (2005) el neoliberalismo como modelo predominante en el mundo actual, es un proyecto político integral de organización económica y social, con una concepción de cómo satisfacer las necesidades 
sociales. Se basa en la libertad de mercado, generación de riqueza, achicamiento del Estado, desmantelamiento del Estado benefactor, disminución de servicios sociales, competitividad a nivel global, beneficios para el capital internacional, mano de obra barata, pérdida de derechos y beneficios laborales y drástica reducción de beneficios sociales. Detrás de la privatización neoliberal de beneficios y servicios sociales, está el objetivo económico de abrir un nuevo campo de acumulación (Ander-Egg, 1995). Colón (2005) señala que en Puerto Rico se profundiza la desigualdad, con la percepción de que dos mundos paralelos coexisten en el pais y se deterioran las posibilidades de las instituciones. La desigualdad social se profundiza en todo el mundo a pasos agigantados.

Las políticas y servicios educativos, no han estado exentas de esta disminución del poder del Estado para cumplir con su responsabilidad social. Más bien, se desarrollan en su diseño e implantación, contradicciones entre el garantizar los derechos y el suplir la demanda del mercado. "La ideología neoliberal tiende a reproducir, más que aliviar las inequidades en las escuelas, a través de políticas y prácticas pedagógicas fundamentadas en el mercado, de reformas curriculares y de rendición de cuentas, así como de requisitos de reclutamiento y admisión de estudiantes" (Macris, 2011, p. 25). De esta manera, el sistema de educación pública termina reproduciendo internamente, la desigualdad inherente a la sociedad de consumo, entre escuelas, personal y comunidades de estudiantes.

Desde fines del pasado siglo, la economía neoliberal ha influenciado las políticas educativas en el mundo. Torres (2005) plantea que en nuestros países latinoamericanos "la educación por sí sola, incluso en el nivel superior, ya no garantiza movilidad social, bienestar ni acceso al trabajo" (p. 14). Por lo que la desigualdad educativa genera a largo plazo mayor desigualdad social. Por otro lado, Bonilla (2004) señala que el sistema educativo público de hoy ha perdido su función civilizadora pero no la socializadora. Añade que "socializa para la sociedad de mercado, sin intentar alterar la concepción filosófica de la escuela republicana" (p. 96). La educación así concebida contribuye al estancamiento social, donde el mercado establece diferencias en la educación de acuerdo con sus propios intereses. Estos se privilegian por encima de los intereses y derechos sociales. La AECF (2008) reconoce la desigualdad social en que vive gran 
parte de la niñez en los Estados Unidos, con el planteamiento de que la nación no puede asegurarle a toda ella, la oportunidad de convertirse en miembros productivos de la sociedad. La educación asi concebida, es más bien objeto de consumo dentro del mercado de servicios, sin otra mística que la de suplir las necesidades presentes y futuras del mercado.

\section{Trasfondo de las políticas educativas que atienden la desigualdad en Puerto Rico}

Las políticas educativas en Puerto Rico han estado históricamente atadas a nuestro proceso de colonización. Hernández (2008) plantea que desde los inicios del siglo XX y bajo la colonización norteamericana en Puerto Rico, la educación se utilizó como instrumento para el proyecto ideológico de americanización de nuestra sociedad y de base para la expansión del capital norteamericano en nuestro suelo. "En 1928 se iniciaron las segundas unidades rurales o escuelas agrícolas industriales en las zonas rurales para adaptar de manera práctica, la educación del campesinado a su medioambiente rural" (Ortiz, 2000, p. 114). Bourne y Ramos (según citado en Ortiz, 2000) concluyeron que las dificultades de la mayoría de la población para acceder a un mejor nivel de vida, no era un problema exclusivo de estilos de vida, sino también de la desigualdad resultante de la economía. Se trata de un reconocimiento de la desigualdad social desde muy temprano en la relación entre los Estados Unidos y Puerto Rico.

Burgos (1998) señala que las pioneras del Trabajo Social en Puerto Rico, estuvieron entre las profesionales formadas y destacadas para trabajar en las segundas unidades rurales, mejorando los niveles de vida en comunidades de extrema pobreza, con problemas de salud y analfabetismo. La pobreza es también señalada por Colón (2005), al plantear que en la década de 1930 había pobreza en más del $80 \%$ de los hogares, una tasa de desempleo de $50 \%$, una tasa de analfabetismo de $41.4 \%$; y enfermedades como tuberculosis, malaria y desnutrición. Además, hubo una reducción de salarios en un $35 \%$ y del ingreso per cápita en $30 \%$. La pobreza extrema y las necesidades crecientes en la década de 1930, llevaron al presidente de Estados Unidos, Franklin D. Roosevelt, a impulsar el Nuevo Trato, como un conjunto de políticas sociales para atender el desempleo y la pobreza. Se creó la Emergency Relief Administration en 1933, extensiva a Puerto Rico, para la inversión en proyectos de infraestructura, alimentación, salud, educación y adiestramiento. 
De acuerdo con el DE (2012), en esa época, además de la construcción de escuelas, se promovió la educación primaria y la alfabetización de adultos.

Luego de la Segunda Guerra Mundial e inicios de la segunda mitad de siglo XX, Puerto Rico comenzó a dar pasos agigantados en materia de educación. Se inició un proceso de desarrollo social, en términos del derecho a educación gratuita hasta el nivel secundario para toda la niñez y su acceso a educación superior. En la segunda mitad del siglo XX, nuestro sistema educativo público, incluido el nivel superior, tuvo un gran crecimiento e importantes logros. La década de 1970, bajo la dirección del Dr. Ángel Quintero Alfaro, se conoce como la década de la educación en Puerto Rico, por el impulso a la educación y el desarrollo de modelos escolares de calidad para toda la niñez. Esto propició la especialización y profesionalización de la fuerza trabajadora, la movilidad social para amplios sectores de la población; y la alfabetización de adultos, principalmente en las comunidades rurales. Para la década de 1980 , se había alcanzado $90 \%$ de alfabetización y $80 \%$ de la niñez en edad escolar se encontraba matriculada en la escuela (Ley 149, 1999, pp. 2-3). Hubo avances significativos en matrícula, instalaciones físicas, ofrecimientos curriculares, recursos humanos, asignación presupuestaria y servicios. Sin embargo, en Puerto Rico también se dieron grandes y complejas transformaciones sociales, que de manera muy acelerada han impactado al sistema de educación pública.

Para la década de 1980, ya se hacían señalamientos sobre la calidad de la enseñanza, el exceso de burocratización, y la falta de eficiencia en los procesos administrativos y de toma de decisiones; así como de desigualdad entre escuelas en el ofrecimiento y en la calidad de los servicios. En el decenio de 1990 en Puerto Rico se hacía referencia a un sistema educativo público con un crecimiento acelerado y en función de la economía de mercado. EI DE no respondía de manera adecuada a las necesidades y exigencias de los diversos sectores de la población escolar, ni se atemperaba de manera equitativa e inclusiva, a los rápidos cambios sociales. En el año 1993 se aprobó la Ley 18 de Reforma Educativa, basada en el concepto de escuelas de la comunidad, como aspiración y concreción de mayor autonomía escolar. Con un ambicioso proyecto de reforma y con algunos logros, esta ley confrontó problemas en su articulación y escándalos fiscales en su implantación. 
En 1999 se aprueba la Ley Número 149, Ley Orgánica del Departamento de Educación. La Ley 149, según enmendada, dispone una política pública basada en los siguientes principios: la excelencia como aspiración fundamental del sistema educativo; el estudiante como centro del sistema y el maestro como agente de cambio constructivo en el sistema; la autonomía de la escuela como medio necesario para la eficiencia del sistema; el fortalecimiento de la educación vocacional, técnica y de altas destrezas; y la participación ciudadana como necesidad del proceso educativo. Esta ley persigue la autonomía como principio esencial y concibe a la escuela como "un ente dinámico, con capacidad para acoplar sus ofrecimientos a las necesidades de los alumnos" (1999, Art. 102). Sin embargo, aunque contiene elementos importantes y necesarios para el mejoramiento de la educación pública, requiere para su fortalecimiento y real autonomía, de mayor descentralización en la toma de decisiones y democratización en los procesos.

El reto actual de la Ley 149 es atemperar el sistema educativo público a los rápidos cambios que acontecen en el Puerto Rico del siglo XXI, con el estudiantado y el magisterio como centro y con una real participación ciudadana. Se necesita poner la educación al alcance de todos los sectores, armonizando las metas educacionales con los objetivos de nuestro país. Sin embargo, en su implantación, las acciones han ido dirigidas hacia su fragmentación mediante cartas circulares dirigidas al cumplimiento con aspectos específicos de políticas educativas federales. Las cartas circulares son el mecanismo que establece el DE para regular la implantación de su política pública.

Para algunos sectores de la población escolar, la labor del DE ha sido efectiva. No obstante, a la escuela puertorriqueña le ha resultado particularmente difícil apoyar el desarrollo de sectores significativos de la población estudiantil. Esos sectores del estudiantado reflejan índices más bajos de aprovechamiento académico, una alta incidencia de fracasos y deserción escolar. El Informe de Transición (2004) del DE, estimó en 34\% del estudiantado que no completa la escuela superior.

Quintero (1996) señala entre las principales críticas al sistema educativo de Puerto Rico las siguientes: un currículo escolar poco pertinente, mala comunicación entre los diversos sectores vinculados a la educación, carencias en la formación del personal docente, así como en la administración y una 
planta física deteriorada. El sistema educativo público ha sido altamente criticado por la calidad de la enseñanza y por ser considerado como uno burocrático y muy centralizado. Los entes coprotagonistas del sistema educativo bajo la Ley 149, han sufrido una disminución real, al igual que en su valoración. El Perfil de Escuelas Públicas de Puerto Rico, refleja que entre el año escolar 2008-2009 y el 2012-2013, la cantidad de docentes disminuyó en 6,126 y la matrícula estudiantil en 56,219.

\section{Las políticas educativas federales en el siglo XXI}

Actualmente estamos ante uno de los momentos de mayor dominación ideológica del gobierno federal sobre el sistema educativo de Puerto Rico. A partir del 2002, entra en vigor la ley federal No Child Left BehindAct (NCLB), cuyas metas son: el desarrollo de calidad en la dimensión académica, personal y social del estudiante; recursos humanos altamente cualificados; participación efectiva de madres y padres, las comunidades y otras instancias públicas, privadas y del tercer sector en el proceso educativo; condiciones óptimas para el aprendizaje; y fortalecimiento institucional en los procesos administrativos y fiscales.

NCLB parte del reconocimiento de acceso limitado y calidad desigual en los servicios educativos para toda la niñez. Sin embargo habría que preguntarse ¿qué cambios propone NCLB en el alcance de los servicios educativos a mediano y a largo plazo? Kosol (2005) señala que actualmente Estados Unidos se encuentra ante un nuevo sistema educativo de apartheid, en el cual la población estudiantil pobre y de minorías étnicas se encuentra segregada en escuelas con problemas de bajo rendimiento académico, de limitaciones en estructura física y organizativa, así como en presupuesto y liderazgo. Esto significa que los resultados en la implantación de dicha ley han desvirtuado sus propósitos originales de reducir la desigualdad educativa en los sectores pobres del estudiantado.

NCLB ha fracasado en su intento de dar educación de calidad a toda la niñez por varias razones. Primero, privilegia los resultados de las pruebas estandarizadas por encima del proceso de enseñanza - aprendizaje, lo que pone al proceso medular de la educación, en función del resultado en unas pruebas. En entrevistas informales sostenidas con personal escolar, se alega que para la administración de las pruebas en el pasado, en algunas escuelas se desalentó la participación del estudiantado 
inmigrante, con rezago académico, de educación especial, con problemas del comportamiento o violencia, así como de quienes se encontraban en riesgo de dejar la escuela. Incluso, se llegó a detectar fraude en la administración de las mismas, para obtener resultados favorables a la escuela.

La NCLB desvaloriza la labor del magisterio achacándoles la responsabilidad por el desempeño estudiantil, sin tomar en cuenta los problemas organizacionales, las necesidades particulares, así como el contexto cultural de las escuelas y las comunidades que atienden. Rivas (2008) plantea que la NCLB obvia la realidad de Puerto Rico y de las minorías étnicas en los Estados Unidos y le impone al magisterio la responsabilidad por el desempeño estudiantil, sin considerar factores como la pobreza y la marginación. Esa ley privilegia el método científico como único método de enseñanza, así como la eficiencia y no el aprendizaje, como indicador de logro. NCLB pone énfasis a las ciencias y matemáticas sobre las humanidades y ciencias sociales (Becker, 2008 , p. 25). Contradictoriamente pasa por alto la contribución de las escuelas universitarias de formación de docentes, dando prioridad a certificar a docentes como high qualified teachers, a través de cursos cortos en áreas especializadas. En Estados Unidos, aún con la implantación del Título I, la desigualdad educativa ha sido creciente y en detrimento de estudiantes provenientes de minorías étnicas como las afroamericanas e hispanas (Kosol, 2005). Los fondos para las escuelas se condicionan al desempeño de sus estudiantes, en las pruebas desarrolladas con criterios establecidos por el gobierno federal, bajo los principios de eficiencia del neoliberalismo.

Con el reconocimiento del presidente Barack Obama de la desigualdad en el sistema educativo, se establece el Plan para la Reforma de la Escuela Primaria y Secundaria en los Estados Unidos (ED, 2010). El plan contempla la integración de las reformas en el área educativa de la American Recovery and Reinvestment Act (ARRA) del 2009, la Ley NCLB del 2001 y el Programa Título I, Elementary and Secondary Education Act., Parte A Improving the Academic Achievement of the Disadvantaged. El Plan de Reforma establece cinco prioridades: estudiantes listos para la universidad y el trabajo; excelentes maestros y líderes; elevar el nivel y premiar la excelencia; equidad y oportunidad; y promover la innovación. "No podremos mantener la promesa norteamericana de igualdad de oportunidad si no somos capaces 
de proporcionar una educación de primera clase a todos los niños", concluye Obama (ED, 2010, p. 5). La implantación de ESEA no ha podido reducir la desigualdad educativa, sino que ésta se ha acrecentado.

\section{Impacto de la política ESEA en la niñez pobre}

La Ley de Educación Elemental y Secundaria, en inglés, Elementary and Secondary Education Act (ESEA), de 1965, se estableció con el Título I dirigido a facilitar a todo el estudiantado, igual oportunidad de recibir una educación de la más alta calidad posible ( Burch, 2007). El Título I provee ayuda financiera a escuelas en áreas geográficas en las que se concentran familias con bajos ingresos económicos. Además, auspicia programas que contribuyen a alcanzar las necesidades especiales de la niñez en desventaja educativa y aumentar el nivel de desempeño académico.

Desde la segunda mitad del siglo pasado, Puerto Rico ha recibido los fondos Título I en todas las escuelas, ya que los criterios de elegibilidad son: alto nivel de pobreza, bajo desempeño académico y provisión de servicios alimentarios en la escuela. ESEA debe impactar aproximadamente al $80 \%$ de la matrícula de estudiantes del sistema público por medio de los servicios educativos suplementarios. Sin embargo, su implantación ha sido señalada en varios momentos con deficiencias tanto en el manejo de los fondos como en los resultados obtenidos por las escuelas, en el aprovechamiento académico de sus estudiantes y en el aumento en la deserción escolar.

Los servicios bajo ESEA o servicios educativos suplementarios (SES) consisten de tutorías y otros servicios de enriquecimiento académico, mayormente en lectura, expresión del lenguaje y matemáticas (DE, 2011). EI DE es responsable de que estén disponibles para el estudiantado elegible, de monitorear su implantación; y de evaluar la calidad y efectividad de los servicios de las compañías contratadas como proveedores [Sección 1116 (e) (4) (D); 34 C.F.R. § 200.47 (a) (4)]. Entre sus responsabilidades, el DE debe no sólo hacer pública la lista de proveedores sino también los informes sobre el cumplimiento y los estándares de evaluación de los SES. Para el año 2005-2006, se estimaba en $82 \%$ el porciento promedio de estudiantes bajo el nivel de pobreza en Puerto Rico (CRH Consulting, 2008). De acuerdo con el Education Department Data Express, en el año académico 2009-2010, Puerto Rico contaba con 1,495 escuelas 
públicas y 493,393 estudiantes, de los cuales el $91.4 \%$ se encontraban bajo el nivel de pobreza. El $100 \%$ de las escuelas participan de los SES porque cuentan con servicios de alimentos de comedor escolar. Sin embargo, estudios indican que existen diferencias entre la elegibilidad del estudiantado y la matrícula o asistencia a los servicios. La población estudiantil que más se ausenta a clases y más necesitada de los SES, es más propensa a no matricularse, a dejar de asistir y a no completar el programa de servicios (Burch, 2007).

Desde el año 2006-2007 hasta el 2012-2013, Puerto Rico ha recibido en fondos de ESEA $\$ 3,507,728,990$, otorgados a compañías privadas para ofrecer los SES, con $\$ 29,642,003$ de aumento en dicho período. Por el resultado de las Pruebas Puertorriqueñas de Aprovechamiento Académico, criterio importante para medir la efectividad de los SES, no se observó mejoramiento en el desempeño del estudiantado ni en el de las escuelas, a pesar del aumento en presupuesto en la última década para la prestación de esos servicios. Por el contrario, se observa una relación inversa entre el presupuesto invertido por la ESEA, los resultados proficientes y avanzados obtenidos por los estudiantes en las Pruebas y las escuelas en plan de mejoramiento durante los últimos siete años. (Ver Tabla 1).

Las escuelas entran en plan de mejoramiento cuando no cumplen con los requisitos de desempeño escolar conocido como Índice de Progreso Anual Adecuado. El 45\% de esas escuelas llevan 5 años o más en plan de mejoramiento y atienden al $75 \%$ de la matrícula estudiantil (Sapientis, 2011). "Para el 2005-2006, todas las escuelas con 6 años o más en plan de mejoramiento, atendían a una matrícula con $80 \%$ o más del estudiantado, bajo el nivel de pobreza" (CHR Consulting, 2008, p. 13). Lo anterior significa que el estudiantado que vive en extrema pobreza estudia en escuelas que están en plan de mejoramiento.

Para lograr la meta de ESEA, de atender la desigualdad educativa, el DE debe exigir de parte de las compañías contratadas, mayor calidad y excelencia, así como la rendición de cuentas y transparencia en sus servicios y procesos administrativos. Durante el año 2010-2011, unas 31 compañías tuvieron contratos con el DE por la cantidad aproximada de 107 millones de dólares (Cámara de Representantes, P. 3558, 2011). Si esa práctica no se evalúa con rigurosidad, puede propiciarse la corrupción en la relación circular entre partido-gobierno-empresa. 
Entre los estudiantes servidos por los SES, quienes tienen mayor necesidad, no son atendidos de manera adecuada. Para algunas poblaciones estudiantiles elegibles (educación especial, inmigrantes, con problemas de salud mental o en el sistema de justicia juvenil) que actualmente no reciben los servicios, sus necesidades quedan sin ser atendidas. Nos dice Burch (2007) que "las poblaciones estudiantiles en mayor vulnerabilidad están siendo menos atendidas o quedan fuera de los servicios, de manera desproporcional al resto del estudiantado elegible, en contradicción del propósito de ESEA" (p.5). La práctica de utilizar en una política pública un esquema de mercado, impacta de manera adversa la población estudiantil que por diversas condiciones se encuentra a mayor riesgo de deserción escolar.

\section{Principales retos y recomendaciones en torno a la deserción escolar}

La situación económica que enfrenta el país requiere de medidas que a largo plazo promuevan la protección y el mejoramiento de la calidad de vida de la niñez y la prevención de la deserción escolar. Ya que a pesar de la legislación protectora, la niñez se encuentra actualmente en un estado de mayor vulnerabilidad, en la medida en que aumenta la desigualdad social, la crisis económica, el desempleo y la violencia. Marxuach (2012) advierte que "la transmisión inter-generacional de la desigualdad afecta el crecimiento económico a largo plazo" ( $p$. 11). El garantizar el derecho a una educación de calidad para toda la niñez le compete al Estado y a la sociedad. Puerto Rico tiene urgencia de un proyecto educativo inclusivo, que responda a las necesidades y metas del país. De la atención de las políticas educativas a la desigualdad, dependerá la viabilidad de nuestro futuro como país.

No es una opción ética, el desechar a parte de la población que se supone participe en la construcción futura del país, desde antes que alcance su pleno desarrollo. Este es un reto que implica responsabilidades compartidas entre diversos sectores sociales para proteger el derecho al pleno desarrollo de nuestra niñez, garantizado por la Constitución. El Estado debe recuperar la confianza de la ciudadanía y su legitimidad. Para lograrlo, debe generar procesos inclusivos, transparentes, abiertos a la participación ciudadana y al consenso. Establecer, implantar y evaluar las políticas educativas, basadas en los principios de justicia social, respeto, democracia, cooperación, 
inclusión, participación y equidad. Debe procurar el desarrollo económico sin sacrificar la protección de derechos humanos.

La legislación debe promover el ejercicio y fortalecimiento de una gobernanza adecuada que contribuya a reforzar una real rendición de cuentas, incrementar la participación y acabar con las desigualdades en el ámbito de la educación. La organización United Nations Children Fund (UNICEF, 2011), urge "a priorizar en el análisis del impacto de las políticas sobre la niñez, como estrategia para reducir la desigualdad en el futuro, mediante el modelo Poverty and Social Impact Analyses (PSIA), que implica poner a la niñez en el centro del análisis de políticas públicas" (p. 14). La Organización de las Naciones Unidas para la Educación (UNESCO, 2009) propone "crear sistemas educativos integradores que: amplíen el acceso de los grupos excluidos a la educación; mejoren el entorno del aprendizaje; amplíen los derechos y oportunidades; y elaboren sistemas de acopio de datos" (p. 32). El atender la desigualdad en la niñez a largo plazo representará la elevación del desarrollo social y económico de nuestro país. El movimiento de educación alternativa también es una opción viable para la prevención de la deserción escolar, con el cuidado de no convertirlo en una corriente educativa segregada para el estudiantado de comunidades pobres. Como sociedad, debemos reclamar al gobierno el cumplimiento de su responsabilidad de garantizar los derechos ciudadanos. Nos toca a todos y a todas exigir y poner en práctica acciones de justicia social, equidad, participación, democracia e inclusión, allí donde se imponen el culto al mercado y la ética de la rentabilidad. 


\section{Referencias}

Administración de Corrección. (2012). Perfil del joven transgresor 2011. San Juan, PR: Administración de Instituciones Juveniles.

Ander-Egg, Ezequiel. (1995). Diccionario del Trabajo Social. Buenos Aires. Editorial Lumen. pp. 200-201.

Annie Casey Foundation. (2012). Data snapshot on high-poverty communities. Children living in America's high-poverty communities. Recuperado de http://www.aecf.org

Annie Casey Foundation (2008). Kids Count Data Book: State profiles of child well-being. Baltimore, Maryland. Recuperado de http:/l www.aecf.org

Bauman, Zygmunt.(2005). Trabajo, consumismo y nuevos pobres. (2da ed.). Barcelona. Editorial Gedisa.

Bazdresch, Miguel. (2001). Educación y pobreza: una relación conflictiva. pobreza, desigualdad social y ciudadanía. Los límites de las políticas sociales en América Latina. Buenos Aires: CLACSO. ISBN 950-9231-57-6.

Becker, Chad. (2008). American education discourse: language, values and U.S. federal policy. Journal of Critical Education Policy Studies, 8(1), 409-446.

Benabdelaali, Wail, Hanchane, Said, \& Kamal, Abdelhak (2011). A New Data Set of Educational Inequality in the World, 1950-2010: Gini Index of Education by Age Group. National Authority of Evaluation of Educational System. Higher Education Council. Morocco: LEAD.

Bonilla, Javier. (2004). El desafío educativo contemporáneo: ¿Consolidar la gobernabilidad o definir un nuevo paradigma educativo? Gobernabilidad de los sistemas sducativos en América Latina. Buenos Aires Argentina: IIPE-UNESCO.

Burgos Ortiz, Nilsa M. (1998). Pioneras de la profesión del trabajo social. San Juan, P.R. Publicaciones Puertorriqueñas.

Brusi Gil de Lamadrid, Rima. (2010). "Si te dejas llevar"... universidad, geografía y desigualdad. Cuaderno de Trabajo Carvajal 1. Mayagüez, Puerto Rico.

Burch, Patricia. (2007). Supplemental Educational Services under NCLB: Emergent Evidence and Policy Issues. Great Lakes Center for Education Research \& Practice. Michigan, EEUU. Recuperado de www.greatlekescenter.org. 
Cámara de Representantes de Puerto Rico. Proyecto de la Cámara 3558. 16ta. Asamblea Legislativa. (18 de agosto de 2011) San Juan, Puerto Rico.

Center for Desease Control and Prevention. (2005). Homicides among children and Young adults, Puerto Rico, 1999-2003. U.S. Department of Health and Human Services. Atlanta. Recuperado de www.cdc.gov

CHR Consulting. (2008). Profile of the Puerto Rico public education system. Toward 21th. century educational leadership: profile of K-12 public education in Puerto Rico. Jamestown, Rhode Island: Commissioned by the Senate of Puerto Rico.

Colón Reyes, Linda. (2005). Pobreza en Puerto Rico: Radiografía del Proyecto Americano. San Juan, P. R.: Editorial Luna Nueva.

Departamento de Educación. (2011). Manual de Procedimientos para la Administración de los Servicios Educativos Suplementarios -Título I, Parte A, Subparte 1, Sección 1116 (e) (1). Oficina de Asuntos Federales.

Departamento de Educación (2011). Historia de la educación de adultos. Recuperado de www.de.gobierno.pr

Departamento de Educación (2014). Perfil Escolar de Puerto Rico. Años 2006-2007 a 2012-2013. San Juan:Autor.

Departamento de Educación (2004). Informe de Transición de Gobierno. San Juan:Autor

De Sousa, Boaventura. (2003). Desigualdad, exclusión y globalización: hacia la construcción multicultural de la igualdad y la diferencia. La caída del Angelus Novus: Ensayos para una nueva teoría social. (1ra. ed.). Bogotá C.D., Colombia: Colección En Clave de Sur. ILSA, p. 125-165.

Dieterich, Heinz. (1996). Globalización y educación: La ideología. Cuadernos de Economía, 15(25), 113-140.

ED Data Express. (2009-2010). Data about the elementary and secondary schools in United States. Recuperado de http:www. eddataexpress.ed.gov

Estado Libre Asociado de Puerto Rico. (1952). Constitución del Estado Libre Asociado de Puerto Rico. San Juan: Autor

Hernández, Blanca. (2008). Historias de promesas rotas: La violencia institucional en lasvescuelas públicas y privadas de Puerto Rico desde la vivencia e interpretación socio-educativa de los 
estudiantes y los familiares. San Juan, P. R.: Publicaciones Puertorriqueñas.

Kosol, Jonathan. (2005). City Club Presents Jonathan Kosol. Interview about actual apartheid schooling system in America. The Greater Writers. Nov. 1th.

Ley Orgánica del Departamento de Educación Pública de Puerto Rico. (1999). San Juan, Puerto Rico: ELA

Macris, Vicky. (2011). The ideological conditions for social reproduction. Journal for Critical Education Policy Studies, 9 (1), 19-46.

Marxuach, Sergio. (2012). Desigualdad y el crecimiento económico sustentable. Presentación del Centro para la Nueva Economía, ante la Conferencia Económica Anual, celebrada el 17 de febrero de 2012, en San Juan, Puerto Rico.

Minujin, Alberto. (2012). Making the case for child poverty. Child poverty and inequality: new perspectives. Isabel Ortiz, Louise Moreira Daniels \& Sólrúm Engilbertsdottir (Eds.). New York: Division of Policy and Practice. UNICEF, p. 14-17.

Organización de las Naciones Unidas. (1989). La Convención sobre los Derechos del Niño. Recuperado de www.onu.org

Organización Panamericana de la Salud. (2003). La violencia juvenil. Informe Mundial sobre la Violencia y la Salud. Washington: Autor. p 25-55.

Ortiz, Miguel. (2000). La cinderella de la casa: la cocina como espacio de trabajo: 1900 a 1933. En Tras las huellas del pasado: mosaico de historia de Puerto Rico (siglos $X I X$ y $X X$ ). San Juan, P. R.: Isla Negra Editores.

Quintero, Ana H. (1996). Hacia la escuela que soñamos. San Juan, Puerto Rico: Editorial UPR.

Redmond, Gerry. (2008). Child poverty and child rights: edging towards a definition. Journal of Children and Poverty, 14 (1), 63-84.

Rivas, Myrna. (2008). Situación presente de la educación de personas jóvenes y adultas en Puerto Rico. Centro para la Cooperación Regional de la Educación de Adultos en América Latina y el Caribe. Recuperado de www.tendenciaspr.com.

Rodríguez, Norma. (2012 en proceso de publicación). Pobreza en Puerto Rico: Una mirada desde las experiencias de las personas que habitan sectores empobrecidos. 
Sapientis (2011). El estado actual de las escuelas públicas en Plan de Mejoramiento en Puerto Rico, año escolar 2010-2011. San Juan, Puerto Rico: Autor

The Elementary and Secondary Education Act, No Child Left Behind, [107-110]. Recuperado de: http://www2.ed.gov/policy/elsec/leg/ esea02/index.html

Torres, Rosa María. (2005). 12 tesis para el cambio educativo: justicia educativa y justicia económica. Fe y Alegría: Movimiento de Educación Popular Integral y Promoción Social.

UNESCO. (2009). Informe de Seguimiento de la Educación Para Todos en el Mundo 2009. Resumen: Superar la desigualdad: Por qué es importante la gobernanza. París, Francia: Ediciones UNESCO.

UNICEF. (2011). Resource Pack: On integrating a child focus into PSIA. The World Bank. United Nation Children Funds. Recuperado de http://unicef.org

U.S. Department of Education. (2010). Un plan para la reforma: la renovación de la Ley de Educación Primaria y Secundaria (en español). Recuperado de: http://www.ed.gov

U.S. Department of Education. (2014). Funds for State Formula and Selected Student Aid Programs. Recuperado de http:// www.2.ed.gov./about/overview/budget/state

U.S. Census Bureau. (2013). Puerto Rico Community Survey (PRCS 2012). Washington: Author. 


\section{Apéndice}

\section{Tabla 1}

Presupuesto de ESEA, Resultados de Pruebas Puertorriqueñas de Aprovechamiento Académico y Escuelas en Plan de Mejoramiento: Años 2006-2007 al 2012-2013.

\begin{tabular}{cccc}
\hline Año & Presupuesto & $\begin{array}{c}\% \text { Resultados } \\
\text { proficiente y } \\
\text { alcanzados }\end{array}$ & $\begin{array}{c}\% \text { Escuelas } \\
\text { en plan de } \\
\text { mejoramiento }\end{array}$ \\
\hline $2006-2007$ & $\$ 451,344,832$ & $59 \%$ & $47 \%$ \\
$2007-2008$ & $\$ 455,589,077$ & $55 \%$ & $48 \%$ \\
$2008-2009$ & $\$ 510,525,233$ & $36 \%$ & $67 \%$ \\
$2009-2010$ & $\$ 534,235,622$ & $37 \%$ & $86 \%$ \\
$2010-2011$ & $\$ 554,910,386$ & $40 \%$ & $87 \%$ \\
$2011-2012$ & $\$ 520,137,005$ & $41 \%$ & $91 \%$ \\
$2012-2013$ & $\$ 480,986,835$ & $41 \%$ & $89 \%$ \\
\hline
\end{tabular}

Fuente: U.S. Department of Education. Funds for State Formula and Selected Student Aid Programs. Departamento de Educación de Puerto Rico. Perfil Escolar del Departamento de Educación de Puerto Rico.

Presupuesto de ESEA, Resultados de Pruebas Puertorriqueñas de Aprovechamiento Académico y Escuelas en Plan de Mejoramiento: Años 2006-2007 al 2012-2013.

\begin{tabular}{cccc}
\hline Año & Presupuesto & $\begin{array}{c}\text { \% Resultados } \\
\text { proficiente y } \\
\text { alcanzados }\end{array}$ & $\begin{array}{c}\% \text { Escuelas } \\
\text { en plan de } \\
\text { mejoramiento }\end{array}$ \\
\hline $2006-2007$ & $\$ 451,344,832$ & $59 \%$ & $47 \%$ \\
$2007-2008$ & $\$ 455,589,077$ & $55 \%$ & $48 \%$ \\
$2008-2009$ & $\$ 510,525,233$ & $36 \%$ & $67 \%$ \\
$2009-2010$ & $\$ 534,235,622$ & $37 \%$ & $86 \%$ \\
$2010-2011$ & $\$ 554,910,386$ & $40 \%$ & $87 \%$ \\
$2011-2012$ & $\$ 520,137,005$ & $41 \%$ & $91 \%$ \\
$2012-2013$ & $\$ 480,986,835$ & $41 \%$ & $89 \%$ \\
\hline
\end{tabular}

Fuente: U.S. Department of Education. Funds for State Formula and Selected Student Aid Programs. Departamento de Educación de Puerto Rico. Perfil Escolar del Departamento de Educación de Puerto Rico. 
